\title{
An Adaptive Genetic Algorithm-based Background Elimination Model for English Text
}

\section{Tang Xiaohui ( $\nabla$ xiaohui428@163.com)}

Shandong University of Political Science and Law

\section{Research Article}

Keywords: adaptive genetic algorithm, English text, background elimination model

Posted Date: September 21st, 2021

DOI: https://doi.org/10.21203/rs.3.rs-916018/v1

License: (9) This work is licensed under a Creative Commons Attribution 4.0 International License. Read Full License 


\title{
An Adaptive Genetic Algorithm-based Background Elimination Model for English Text
}

\author{
Tang Xiaohui \\ Shandong University of Political Science and Law, Shandong, 250014, China \\ Email: xiaohui428@163.com
}

Abstract: In this paper, an adaptive genetic algorithm is used to conduct an in-depth study and analysis of English text background elimination, and a corresponding model is designed. The curve results after the initial character editorialization are curved and transformed, and the adaptive genetic algorithm is used for the transformation to solve the influence of multiple inflection points of curve images on feature extraction. Then, using the minimum deviation method, the error values of the input characters and the sample set in the spatial coordinate system are calculated, and the deviation values of the angle and the straight line are used to match the characters with the smallest deviation value to match the highest degree. A genetic algorithm is introduced to iterate the feature sets of angles and line segments, and the optimal features are finally derived in the process of cross evolution of generations to improve the recognition accuracy. And the character library is used as input items for average grouping for experiments, and the obtained feature sets are put into the position matrix and compared with the samples in the database one by one. It is found that the improved stroke-structure feature extraction algorithm based on a genetic algorithm can improve the recognition accuracy and better accomplish the recognition task with better results compared to others. Finally, by analyzing the limitations and characteristics of traditional particle swarm optimization algorithm and differential evolution algorithm, and giving full play to the advantages and applicability of different algorithms, a new differential evolution particle swarm algorithm with better performance and more stable performance is proposed. The algorithm is based on the PSO algorithm, and when the population update of the PSO algorithm is stagnant and the search space is limited, the crossover and mutation operations of the DE algorithm are used to perturb the population, increase the diversity of the population, and improve the global optimization ability of the algorithm. The algorithm is tested on a common dataset for text mining to verify the effectiveness and feasibility of the algorithm.

Keywords: adaptive genetic algorithm; English text; background elimination model

\section{Introduction}

With the rapid growth of Internet users and the popularity of various mobile terminal devices, people can easily and quickly publish information on various platforms and devices, which makes data in various forms grow exponentially in various fields and the total amount of data increases dramatically. The value of data resources is increasingly valued today and has become an important strategic resource concerning productivity and cost [1]. Obtaining hidden information with regularity from massive data has also become an important way to improve enterprise productivity and enhance user experience. In the past, enterprises kept scattered data with expensive storage hardware and a single domain, and the way to obtain knowledge through manual search and organization was not only time-consuming and inefficient but also error-prone. Due to the limitations of data organization and traditional data analysis tools, people's needs for data are limited to simple operations such as query and mathematical statistics, and they cannot analyze data at a deeper level to meet the quantitative analysis needs of specific targets. With the dramatic reduction of storage hardware cost and the rapid increase of processor computing power, data acquisition, preservation, and calculation no longer become obstacles, and the demand for higher-level and regular information from massive data has driven the development of data analysis technology [2]. When someone impersonates another person's identity for signature, the victim usually only authenticates the forged signature to obtain evidence after suffering a loss, and it is difficult to authenticate in advance to avoid the loss. The use of offline signature authentication algorithms can use computers to automatically authenticate many handwritten signature images, thus reducing or even replacing the work of human handwriting experts and improving the efficiency of signature authentication. In this way, acts such as signature impersonation can be prevented by active verification, providing higher security for people's daily life.

The complex environmental information, text content, and the diversity of image shooting angles lead to the problem of inaccurate localization in most text detection methods, and even the mature OCR technology cannot achieve satisfactory detection results [3]. In summary, given the difficulties faced in extracting text from natural scenes and the broad application prospects and economic value of this technology, this paper conducts an in-depth study of natural scene text detection technology. And for the above problems, many solutions have 
been proposed by researchers in recent years. Before that, structural feature extraction and statistical feature extraction were also proposed to extract multiple feature vectors to achieve the best recognition effect. However, due to the different styles of fonts and different writing habits, it is impossible to unify the writing styles. Therefore, the difficulty of the current research is still how to refine the feature extraction, maximize the same set for matching, and improve the recognition accuracy [4]. In the analysis of the factors influencing the difficulty of English reading comprehension, ANOVA and correlation analysis were used to eliminate the factors that did not have a significant influence on the difficulty; in the process of calculating the weight values of each influencing factor of difficulty, the data of experts judging the importance degree of each factor were organized and calculated using hierarchical analysis to derive the weight values of each influencing factor; in addition, in the verification of the reliability and validity of the adaptive testing system. The correlation analysis was conducted between the test data of the pre and post-test levels of English reading comprehension ability, and the subjects' English test scores.

Therefore, this study further verifies the applicability of item response theory in English reading; in addition, current research on the definition method of English reading comprehension difficulty is even less common, and this study makes up for the lack of research in this area and provides reference and reference for subsequent researchers. At the level of practical significance, based on the developed adaptive testing system, the test and application of English reading comprehension ability were conducted in the junior English teaching classroom, and the specific testing process was combined to provide an implementation path with a reference value for adaptive testing of English reading comprehension ability. In estimating the difficulty parameters of the items, this study invited experts working in English research to judge the importance of the factors influencing reading comprehension difficulty, and the experts determined the relative importance of each factor based on their professional knowledge and work experience. Finally, at the end of the adaptive test, data on the subjects' satisfaction with the test, the convenience of the operation process, the friendliness of the operation interface, and the subjects' suggestions for the improvement of this test system were collected by the questionnaire method.

\section{Current status of research}

Connected domain-based methods use a bottom-up strategy to aggregate regions into connected regions based on the characteristic of text with specific similar features, then filter non-text regions using trained classifiers or heuristic rules, and finally fuse adjacent individual texts to form text lines [5]. The connected domain-based methods can be further divided into edge-based detection methods and text-level detection methods. The edge-based text detection method first extracts information such as edges and corner points of text in an image, then obtains the connected regions as candidate regions by smoothing and morphological operations, and finally verifies the candidate regions by using heuristic rules or classifiers to filter out non-text connected domains [6]. This method can quickly extract the text in the image, but for the text with shadows or uneven illumination, the detection effect is poor because the edges or corner points of the text cannot be detected accurately. Puri et al. proposed a classification-based text detection algorithm for natural scenes based on the idea of sparse representation of distinguished dictionaries [7]. The algorithm first detects image edges by wavelet transform while sliding window scans the detected image edges as patches, then obtains text candidate regions by a simple classification process using two learned discriminative dictionaries, and finally uses adaptive tour smoothing algorithm and contour projection analysis to further fine filter the candidate regions to form stable text regions [8].

K-means is a classical division-based unsupervised clustering algorithm with the advantages of simplicity, efficiency, and ease of implementation, and has been applied in many fields [9]. The algorithm starts with random initialization of a set of cluster centres and then repeats the steps of dividing the data set using the cluster centers and updating the cluster centers within the divided set of clusters until the cluster centers converge [10]. Abdel-Kader divides the population into two subpopulations based on the size of individual fitness values and then updates the PSO algorithm and genetic algorithm in each of the two subpopulations. Pandey proposed a dynamic differential evolutionary algorithm for clustering that uses the median value of random samples to initialize the cluster centers and divides subpopulations for updating and then merging [11]. Since a single evolutionary algorithm cannot have good performance in terms of stability, convergence speed, and searchability, trying to use a hybrid algorithm for clustering becomes a feasible solution [12]. Li et al. 
improved the K-means algorithm using a genetic algorithm to adjust the search direction of the population by changing the variation rate at each iteration and performing variation operations to avoid clustering algorithm from falling into local optimum [13]. Song used randomly selected samples from each cluster set after clustering by genetic algorithm with better global optimization capability as initial values of individual clustering centres and then used a hybrid GQPSO algorithm for clustering by quantum particle swarm optimization algorithm [14].

In a comprehensive view, the current theoretical studies of adaptive test question banks by scholars are relatively rich, giving the implementation and optimization schemes of attribute classification, content balance, and item generation in the process of question bank construction, but there are still more problems in the practical studies of adaptive test question banks, such as quality control of question banks, exposure control of questions, automatic item generation based on the cultural background of subjects, and so on [15]. In addition, there is not much literature on adaptive testing of English reading comprehension, and the parameter values of each item are estimated by statistical analysis of many sample data when constructing an English reading comprehension question bank, and few studies have calculated the item parameters directly for the reading material and the questions themselves. Based on this, this study will use a new method to estimate difficulty parameters when constructing English reading comprehension question banks and provide new ideas for similar difficulty parameter estimation in the future.

\section{Adaptive Genetic Algorithm for English Text Context Elimination Analysis}

\subsection{Improved Adaptive Genetic Algorithm}

The idea of genetic algorithm (GA) comes from biological evolution, where the evolved individuals will partially inherit the good characteristics of their parents, so the practical application optimization problem can be transformed into a genetic problem by direct encoding or indirect encoding, and the optimal solution is sought based on the target adaptation. Multi-generation evolution is the key step of genetic algorithm to find the optimal solution, but the image factors of each evolutionary generation will not adapt according to the results of recent evolution, so the quality of the optimal solution after satisfying the termination condition is relatively poor, and it is difficult to obtain the global optimal solution in complex multi-objective optimization problems [16]. To solve this problem, scholars have proposed the adaptive genetic algorithm (AGA), which can be used to improve the parameter setting problem in the algorithm and make adaptive designs for the crossover probability and variation probability formulas, so that the crossover and variation processes change with the overall adaptation and evolutionary generations to find a more optimal solution in the limited local search space.

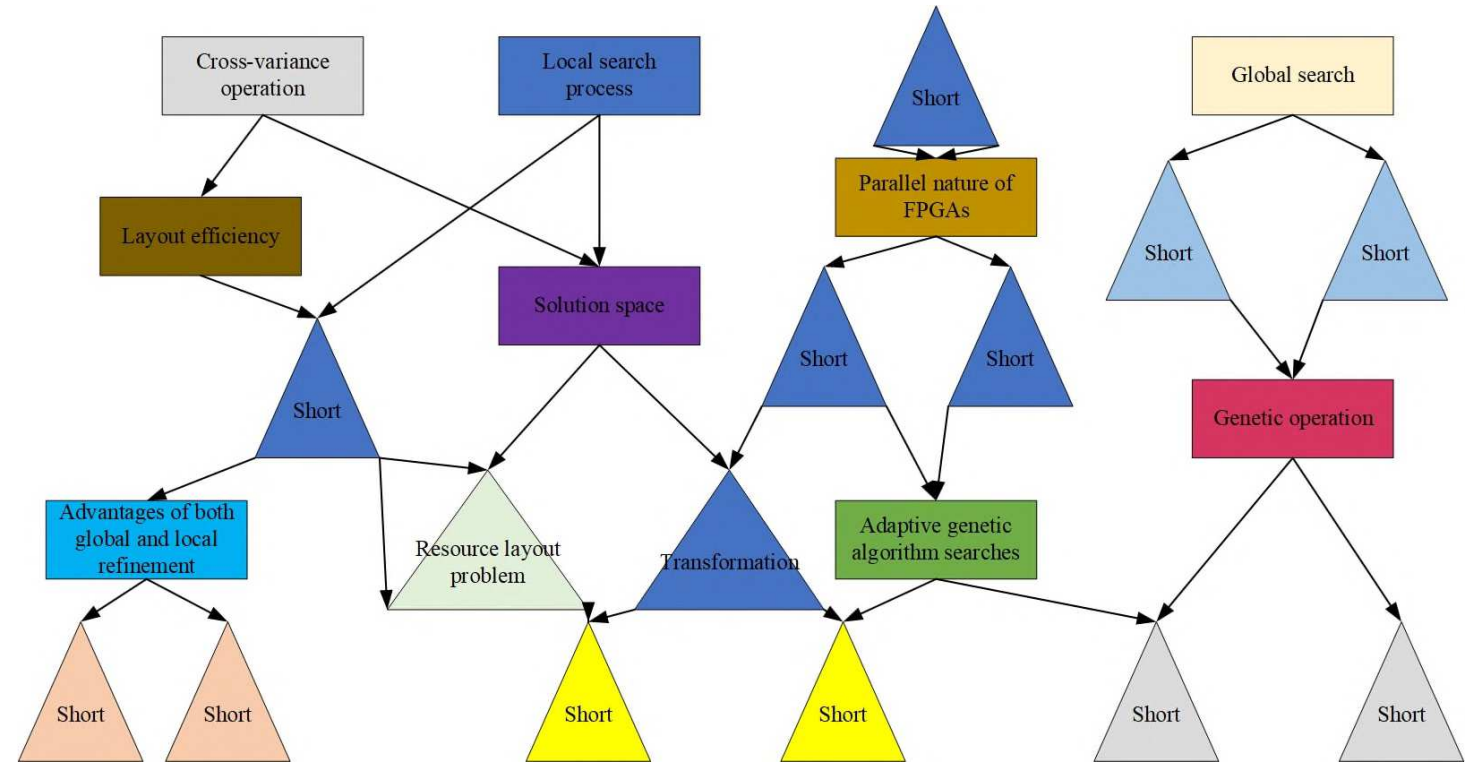

Figure 1 Improved adaptive genetic algorithm framework

The adaptive genetic algorithm searches the solution space based on the adaptive cross-variance operation, which can refine the local search process while global search, and has the advantages of both global and local refinement. In addition, since the adaptive genetic algorithm can perform genetic operations based on population grouping, many steps can be carried out in parallel, which can effectively reduce system pressure and improve 
layout efficiency [17]. Due to the parallel nature of FPGAs and the advantages of heuristic layout, the adaptive genetic algorithm has strong applicability to FPGA resource layout problems and has been widely used in FPGA design-related research in recent years.

In adaptive genetic algorithms, the feasible solution of a multi-objective optimization problem is transformed in some way for algorithmic manipulation, and this transformation method is called coding. This transformation is to convert the solution from its solution space to the encoding space to obtain the mapping relationship between the solution space and the encoding space, as shown in Figure 1.

Adaptive genetic algorithms use fitness to represent the degree of chromosome excellence and fitness as an indicator to select evolved individuals, so a reasonable and effective fitness function needs to be designed. Equation (1) is a common fitness function that can be used to solve the maximum (minimum) optimization problem [18]. However, this expression may yield negative results, which cannot be applied to roulette strategybased selection algorithms in practical applications, and when the values of certain functions differ greatly, the average value of the results will not represent the overall fitness of the population, and the population will be locally optimal and locally worst, which eventually affects the optimization effect.

$$
\text { Fitness }(f(x))=\left\{\begin{array}{c}
f\left(x^{2}\right) \\
-f\left(x^{2}\right)
\end{array}\right.
$$

To solve the appealing problem, equations (2) and (3) can be used to solve the maximum (minimum) optimization problem. Where mins $(\max )$ are a predetermined number that is appropriately small (large) and is generally taken as the minimum (maximum) function value of the estimated objective function. However, because mine (max) is estimated in advance and is less accurate, the fitness function is still not sensitive enough, which makes the algorithm performance unstable and can be subsequently improved based on the adaptive parameter change approach.

$$
\begin{aligned}
& \text { Fitness }(f(x))=\left\{\begin{array}{c}
f\left(x^{2}\right)+\mathrm{C}_{\text {min }}, f(x)+\mathrm{C}_{\text {min }}>0 \\
0, f(x)+\mathrm{C}_{\text {min }} \leq 0
\end{array}\right. \\
& \text { Fitness }(f(x))=\left\{\begin{array}{c}
C_{\text {max }}+f\left(x^{2}\right), f(x)-\mathrm{C}_{\text {min }}>0 \\
0, f(x)+\mathrm{C}_{\text {min }} \leq 0
\end{array}\right.
\end{aligned}
$$

This algorithm designs a multi-objective optimal fitness function, the results of which rely mainly on the metrics that have been determined for the evaluation of the FPGA resource layout algorithm for the design of the two commonly used evaluation metrics are as follows: used to evaluate the reasonableness of the task placement for the ratio of resources required for the task and the resources allocated for the layout, the most ideal case is a single-task resource utilization of 1 . However, in the case of single-task placement, because the layout algorithm is required to reserve enough resource space for wiring to allocate resources to the task, so the algorithm may generally allocate more than the minimum resources needed by the task. The resource utilization of a particular layout can be expressed as a weighted average of the utilization of a single task, with a larger RE indicating that the resource management algorithm can find space for the task group closer to the needs of the task group itself. The expression for the calculation of RE is as follows.

$$
R E=\frac{\sum_{i=1}^{P} M_{i}^{2}}{\sum_{j=1}^{P} R_{i}^{2}-A_{N}}
$$

Where $\mathrm{p}$ is the total number of tasks, $M$ is the minimum number of resources required for a single task, $R$ is the actual number of allocated resources, and $A_{N}$ is the normalized value of reserved resources such as wiring, which is the average of the layout reservation space obtained by the algorithm processing 100 times of resource layout tasks [19]. The Biggest Resource Area (BRA) is used to indicate the rectangular area where the FPGA can continuously allocate resources under a specific algorithm, such as the quadtree based algorithm, where the Biggest Resource Area is the leaf nodes that are not allocated with resources, and the closely aligned genetic algorithm, where the Biggest Resource Area is the unallocated resource area bounded by all closely aligned tasks. This area does not include the space fragments surrounded by multiple tasks. Combining the 
above two layout optimization metrics, the multi-objective fitness function $F$ is designed, and its expression is shown as follows.

$$
F=c_{1} w_{r} \times R E-c_{2} w_{b} \times B A R(5)
$$

Where $c_{1}$ and $c_{2}$ are the scaling factors used to ensure that the two objectives of resource flexibility, resource utilization, and maximum available margin are normalized to the same order of magnitude. $w_{r}$ and $w_{b}$ are system parameters describing the resource utilization and maximum available margin objectives, which are weighted when performing FPGA resource layout optimization.

The adaptive adjustment of the variation probability Pm uses the variation of two numbers generated by a random function, such as the variation of the time and teacher code in the schedule of a class. The performance of the genetic algorithm is affected by crossover and variation, and the magnitude of the adaptive variation probability is not a fixed value but varies with the crossover probability [20]. These two operations, adaptive crossover, and variation are coordinated with each other to ensure the global search ability of the genetic algorithm to obtain the global optimal solution. After the above process operation, the conflict coping mechanism between populations has been established, but the possibility of new conflict contradiction formation cannot be excluded, so it is necessary to carry out conflict detection and elimination work again.

After executing all the above processes, it is considered to have completed iteration of evolution, which is following the evolutionary law and principle of "survival of the fittest", and for each iteration of evolutionary operation, the fitness value of the children left behind will be a little larger compared with that of their parents. If there is no significant increase or no change in the fitness value of the children at the end of the iteration, it means that the approximate global optimal solution of the problem has appeared. Figure 2 shows the optimized design flow chart of the improved novel adaptive genetic algorithm.

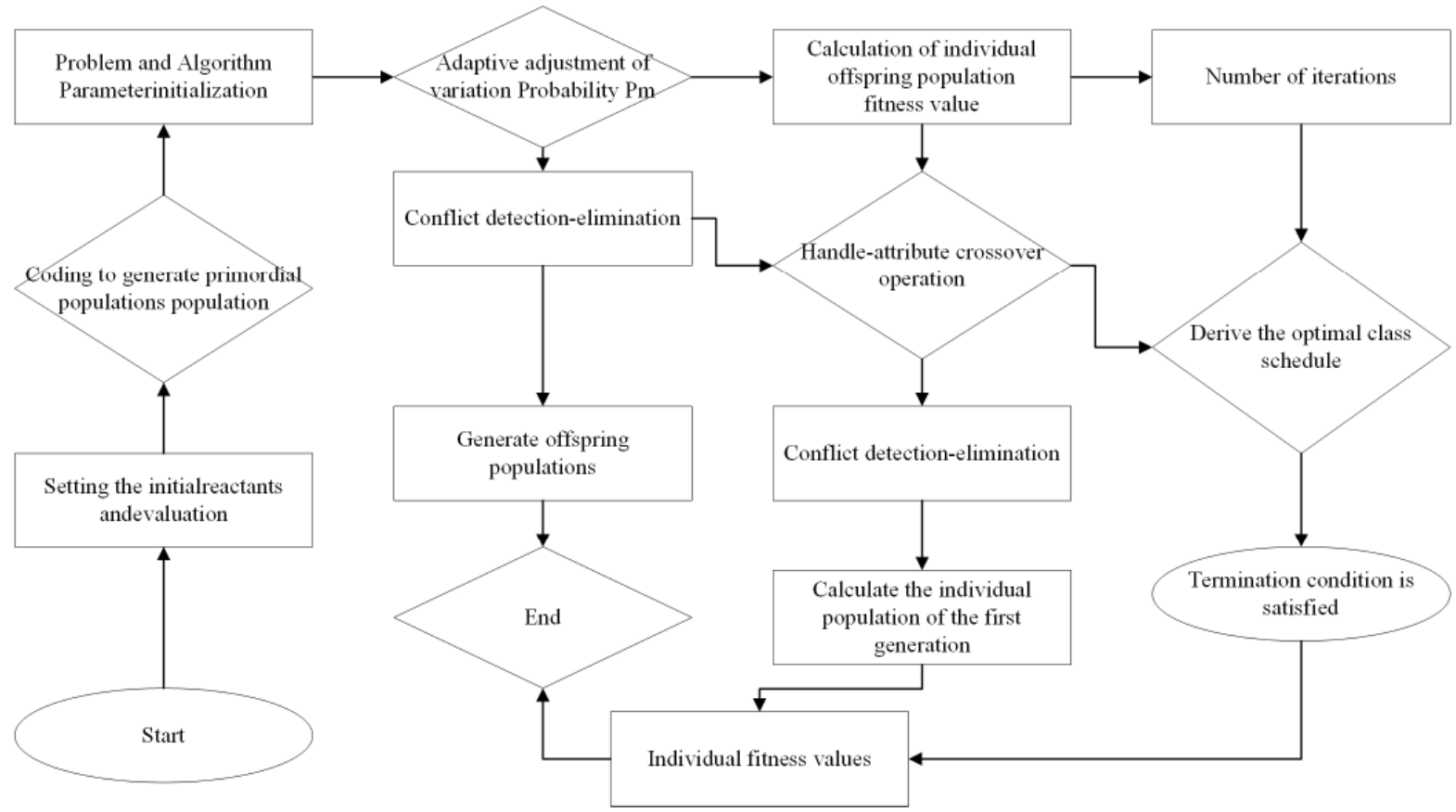

Figure 2 Flow chart of the improved adaptive genetic algorithm

With the adaptation degree already high, the handle-attribute crossover operation designed in this paper can change the chromosome properties locally in the chromosome to further improve the adaptation degree. Based on this, the probability of the handle-attribute crossover operation is adaptively adjusted in this paper.

$$
P_{c}=\left\{\begin{array}{c}
k_{3}^{2}\left(f_{\min }-f\right) /\left(f_{\max }-f_{\text {ave }}\right), f \leq f_{\text {ave }} \\
k_{4}^{2}, f>f_{\text {ave }}
\end{array}\right.
$$

The adaptive genetic algorithm in this algorithm relies more on the role of variation and uses inverse-order variation, in which genes in two different positions are randomly exchanged in the chromosome to be mutated, essentially repositioning the genes in reverse-order arrangement on the vacant positions of the chromosome so 
that the chromosome itself contains tasks in a changed order of arrangement. In the case of a low adaptation, the effect of handle-attribute-based crossover operation will be very limited in enhancing the adaptation, while the reverse order variation can enhance the searchability of the algorithm and improve the adaptation enhancement in changing the order of task arrangement in the chromosome. The probability of variation based on the adaptive algorithm can be calculated as follows.

The word frequency can indicate the degree of contribution of the lexical item to the information expression of this document, and the higher the word frequency, the greater its association with the text. By ranking the word frequency of keywords and then removing feature lexical items with lower frequency, the vector dimension of the text can be reduced to some extent and the computational workload can be reduced. However, in some cases, the less frequent lexical items are more distinguishable and representative, so word frequency is only an important factor in evaluating feature weights.

$$
P_{m}=\left\{\begin{array}{c}
1, f_{\max } \leq T \\
k_{1}^{2}\left(f_{\min }-f\right) /\left(f_{\max }-f_{\text {ave }}\right), f \leq f_{\text {ave }} \\
k_{2}^{2}, f>f_{\text {ave }}
\end{array}\right.
$$

The calculation of lexical item weights should consider not only the frequency of the lexical item in a document but also the distribution of the lexical item in the whole corpus. A lexical item is more representative only if it appears more frequently in a certain document and less frequently in other documents. To fully consider the degree of contribution of both word frequency and inverse document frequency to the expression of text topics, the TF-IDF, the product of word frequency and inverse document frequency, is commonly used as the calculation of word item weights.

$$
T F+I D F_{w}=I F_{w} \times I D F_{w}(8)
$$

At the same time, considering the problems of inconsistent text length and the disparity in the number of dimensions with non-zero weights, Euclidean distance, and Ming's distance cannot correctly represent the similarity between two texts to a large extent, and since the length of the text is normalized in the cosine distance formula, the cosine distance is used to measure the similarity between document vectors, which is more consistent with the characteristics of document vectors with high dimensionality and small feature values.

\subsection{Experimental design for English text background elimination}

In this paper, this method is used for skew correction of signature images. The method achieves tilt correction of the signature image by rotating the minimum inertia axis of the signature curve to coincide with the horizontal direction. After passing the background elimination, the grayscale of the signature stroke in the signature image is unchanged while the background grayscale is reset to 255 . Therefore, the signature stroke curve can be obtained by counting the pixels in the image whose grayscale is not equal to 255 and represented by Equation (9).

$$
C=\left\{S(i)=\left[\begin{array}{l}
x^{2}(i) \\
y^{2}(i)
\end{array}\right], i=1, \ldots, N\right\}
$$

Finally, the angle between the direction of the minimum inertia axis of the signature and the horizontal coordinate axis is the calculated rotation angle [21]. All the pixels in the signature are rotated by this angle around the signature center of mass, and then the skew correction of the signature image is achieved by intercepting the image of the signature stroke part. To effectively eliminate the influence of background on text re-identification, three background elimination methods are designed: pixel-level background elimination method, feature-level unsupervised background elimination method, and feature-level supervised background elimination method. The text image segmentation mask is obtained by the fusion segmentation method in Section 2.2, and then the mask and the text image are fused at the pixel level and the feature level, respectively, by using the MPF (multi-pooling fusion) network as the backbone network.

The feature-level unsupervised background elimination method is to fuse the text segmentation mask with the feature map generated by the network model, using the segmentation mask to retain the feature data of the foreground image of the text and set the background part of the feature data to 0 to achieve the purpose of eliminating the background information of the text. The network structure is shown in Figure 3, firstly, the text 
image is passed through the text segmentation network to obtain the binary segmentation mask, the foreground is 1 and the background is 0 . The new feature map is used to map the feature vector for computing text similarity. The feature set unsupervised background elimination method can reduce the reliance on the accuracy of text mask segmentation. Unlike unsupervised methods, the feature-level supervised background elimination approach makes the network automatically learn to weaken background information [22]. The method adds a network branch and calculates the feature activation loss function; then the feature activation loss function is combined with the text classification loss function as a multi-task learning loss to supervise the network model to extract useful text foreground features.

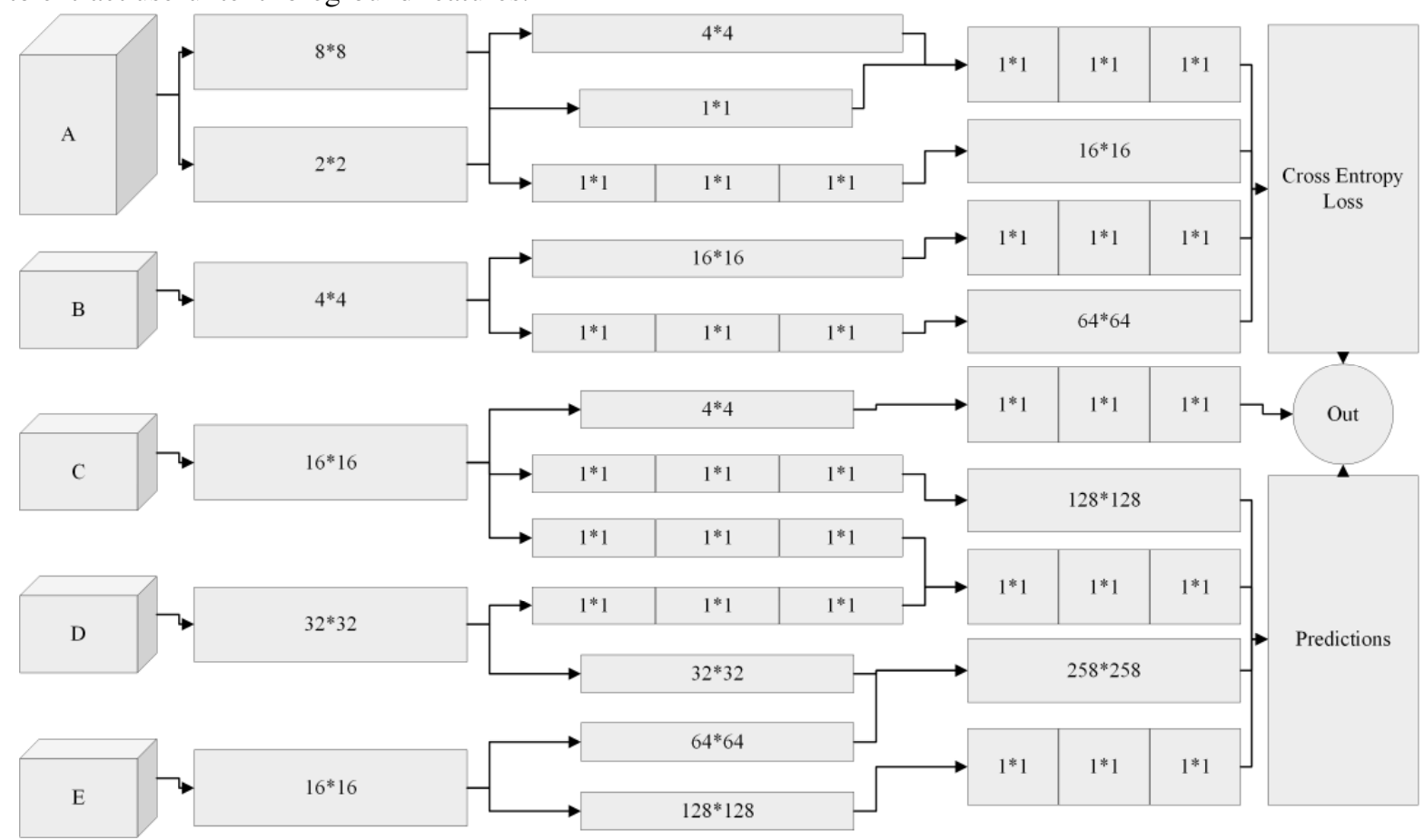

Figure 3 Background elimination network structure

Based on the experimental results, it can be concluded that from the perspective of resource utilization, the quadtree-based layout algorithm, on the other hand, due to its way of mapping resources according to nodes, will have redundant resource occupation, and the resource utilization rate is only $25.6 \%$ in the multi-task layout problem with different resource demands; the two-dimensional random layout algorithm directly maps the resource blocks according to the task size compared to the quadtree-based layout algorithm. However, the resource utilization rate is only $43.2 \%$, which is relatively low because the task layout is too loose and the average reserved resources are larger; while the GA-based layout algorithm and the AGA-based layout algorithm obtain a tighter task layout because of the advantage of heuristic layout, and the resource utilization rate of the layout is also significantly improved, reaching $61.5 \%$ and $61.5 \%$, respectively. The resource utilization of the layout is also significantly improved, reaching $61.5 \%$ and $69.1 \%$, respectively, which can effectively reduce the redundant occupation of resources.

From the perspective of the maximum available blank area, the two-dimensional random layout algorithm is limited because the tasks randomly select blank locations for arbitrary placement, and the resource space is partitioned by each task, and the maximum available blank area is also limited; the quadtree-based resource layout algorithm only has a small portion of blank areas for the deepest remaining nodes due to its logic of allocating resources according to nodes. The genetic-based resource layout algorithm and the adaptive AGAbased resource layout algorithm, on the other hand, have obvious advantages. Utilizing heuristic layout, a tighter layout result can be obtained, and the maximum available blank area reaches $30.8 \%$ and $40.6 \%$ of the resources, respectively, which improves the number of tasks accommodated by the FPGA layout, as shown in Figure 4. 


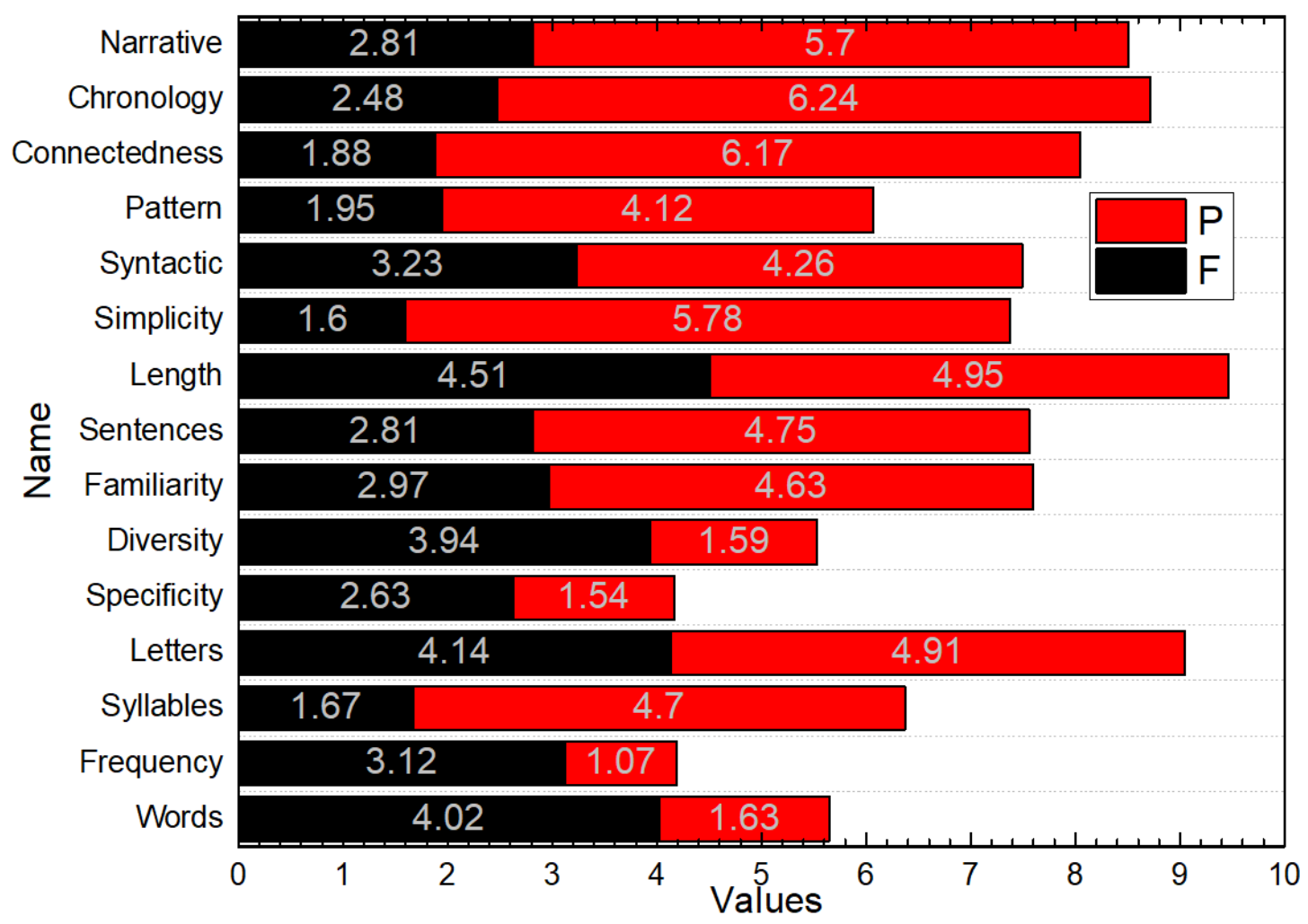

Figure 4 Analysis of variance for each difficulty gradient

From the ANOVA results in Figure 4, the P values of word frequency, a mean number of syllables, mean number of letters, real word familiarity, and narrative are less than 0.001 , indicating that these five factors have an extremely significant effect on the difficulty of reading texts; the P values of word count, lexical diversity, mean sentence length, and syntactic complexity are less than 0.01 , indicating that these four factors have a significant effect on the difficulty of reading texts; the $\mathrm{P}$ values of lexical specificity and $\mathrm{P}$ values for the number of sentences are less than 0.05 , indicating that these two factors affect the difficulty of reading texts [23]. The P values of syntactic simplicity, syntactic type density, connectivity, and temporality were all greater than 0.05 , indicating that these four factors did not have a significant effect on the difficulty of reading materials. Since lexical diversity, syntactic complexity, and syntactic pattern density are considered by multiple indicators, the secondary indicators of these three factors will also be analyzed by ANOVA to test the effect on reading comprehension difficulty. The mean number of words before main verbs and the mean number of modifiers of noun phrases in syntactic complexity are less than 0.01 , which means that they have a significant effect on the difficulty of reading text; only the prepositional phrase density had a value less than 0.05 , but the overall effect of syntactic pattern density on the difficulty of the reading text was not significant.

\section{Analysis of results}

\subsection{Algorithm performance test results}

Test reliability, also known as test reliability, refers to the consistency or stability of the results of a test for the same group of people at different times. In other words, a good measurement instrument must first ensure that the results of multiple measurements remain stable and have certain high reliability to judge test validity, otherwise, the instrument is meaningless. Since the test system in this study is guided by item response theory and uses an adaptive personalized question selection strategy, which makes the subjects' responses vary from test to test, and the IRT has the property of constant estimation of the subjects' ability parameters, the correlation between the subjects' results of these two tests can be regarded as the reliability level of the system when two adaptive tests are conducted within an appropriate time interval. At the end of the two adaptive tests, the two adaptive test scores of each subject were downloaded and counted separately. The mean, standard deviation, maximum and minimum values of the two ability values were first counted, as shown in Figure 5. 


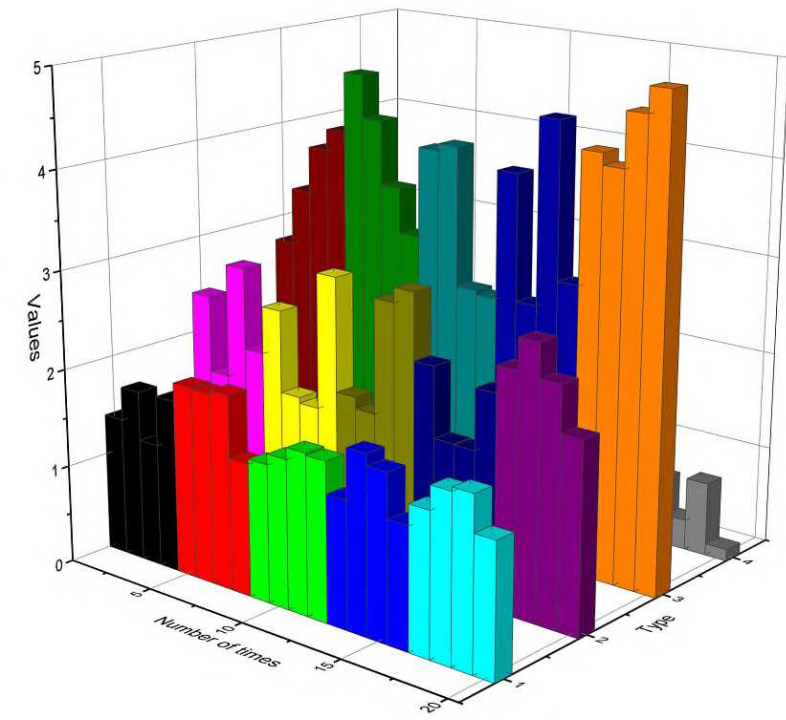

Figure 5 Basic statistics of the two adaptive test scores

The ability estimates of the system range from -3 to 3 . As can be seen from Figure 5 , the mean ability estimates of the two adaptive tests are 0.589 and 0.694 , respectively, which are relatively close to each other, indicating that the subjects' reading comprehension ability levels are relatively consistent within a short period, which means that the adaptive testing system also tends to be stable in its estimation of the subjects' English reading comprehension ability. In addition, the difference between the maximum value and the minimum value is more obvious, which indicates that the system can effectively distinguish the level of ability. As can be seen from Figure 5, the correlation coefficient between the two adaptive test proficiency level values is 0.899 , and the p-value is less than 0.01 , which indicates that the system has good reliability in estimating the English reading comprehension ability in junior high school, and can better reflect the real proficiency level of the subjects.

Through the experiments, the hyperparameters of DenseNet are selected in this paper, and the DenseNet network structure with better performance is obtained. To select a more suitable feature extraction network for offline signature authentication, the classical convolutional neural network structure as a feature extraction network composed of Siamese network is experimented on GPDS dataset in this paper to compare the performance of different structures of convolutional neural networks in offline signature authentication, and the experimental results are shown in Figure 6.

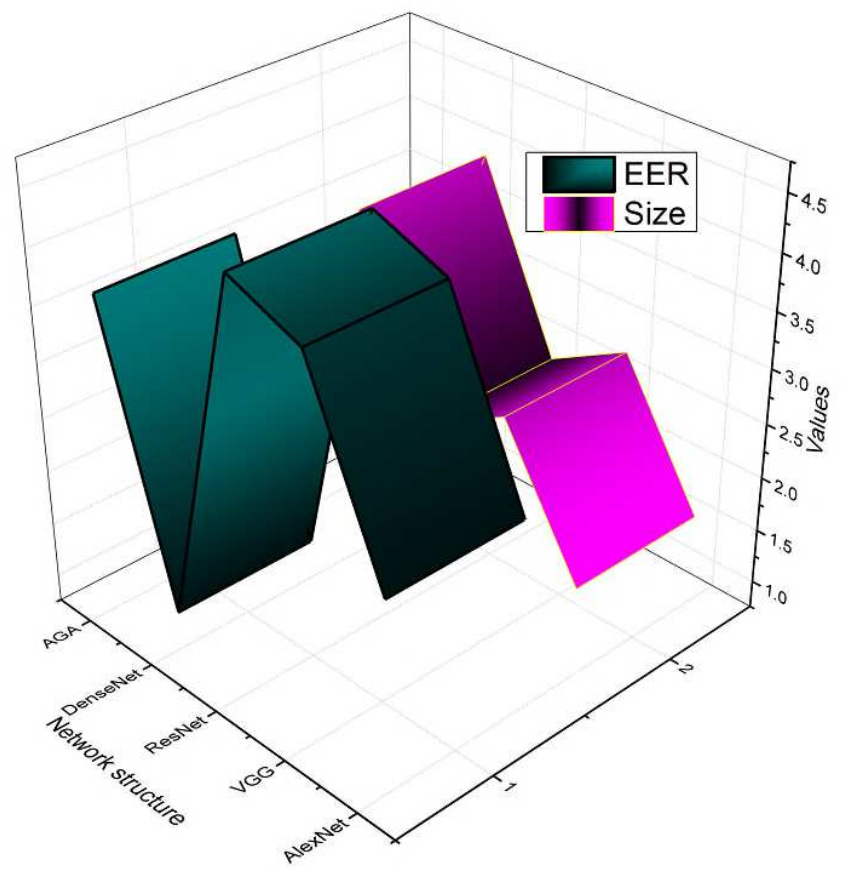

Figure 6 Effect of different structures of feature extraction network on performance 
As can be seen in Figure 6, the DenseNet structure not only achieves higher performance than other convolutional neural network structures, i.e., $10.93 \%$ iso-error rate but also its model size is only $4.2 \mathrm{MB}$. Among them, the VGG network structure achieves similar performance as the DenseNet structure, but the number of parameters in the VGG network structure is more than 3 times that of the DenseNet structure. However, the number of parameters in the VGG network structure is more than three times that of the DenseNet structure, and many parameters easily brings the risk of overfitting. Therefore, in this paper, the DenseNet structure of the offline signature authentication algorithm based on the Siamese network is selected as the feature extraction network for feature extraction of signature images, and the Siamese network is composed for offline signature authentication. Through the experimental comparison in the previous paper, this paper uses the improved Siamese network for similarity metrics and combines it to implement an offline signature authentication algorithm based on region metric fusion. The signature image is regionally divided to obtain seven signature regions, and these signature region subgraphs are substituted for the complete signature image to train the improved Siamese network. In this paper, experiments are conducted on the GPDS dataset, CEDAR dataset, and ChnSig dataset, and each signature region subgraph is fed into the improved Siamese network individually for similarity metric, and the signature authentication is performed based on this to evaluate the performance of the algorithm on different signature regions.

While cosine similarity performs well in areas such as text analysis, it performs poorly in signature authentication. This is because cosine similarity is insensitive to the length of the feature vectors and focuses only on the angle between the feature vectors. The small difference between a real signature and a skilled forged signature makes the angle between the feature vectors vary very little, which is evidenced by the fact that the threshold value of cosine similarity is very close to 1 in the experiments. Although the Euclidean distance performance is better than the cosine similarity, it cannot combine the characteristics of the feature distribution in the high-dimensional feature space, and thus has insufficient performance compared to the metric learning performance. Moreover, the threshold of the Euclidean distance takes the whole negative domain, which will not facilitate the algorithm to adjust the threshold according to the risk. When the Siamese network uses metric learning for feature metrics, it achieves better performance in all data sets. Therefore, in this paper, the metric learning approach is chosen to perform similarity metrics on signature features and then complete offline signature authentication.

\subsection{Analysis of experimental results}

In this section, the candidate region extraction stage makes better use of the color information of the color image based on the improved algorithm in the section and extracts stable polar regions using the improved MSER algorithm on R, G, and B channels, respectively. To compare the performance differences between the improved MSER algorithm for extracting polar regions on three color channels, the classical MSER algorithm for extracting polar regions on grayscale maps. To compare the performance differences of the three methods of the improved MSER algorithm for extracting polar regions on grayscale images, the experiments are conducted on the ICDAR2013 test set using equal MSER thresholds, and the specific experimental results are shown in Figure 7. And in the experiments of this paper, the size of its feature population is set to 5, while the encoding is set to a five-digit binary chromosome to perform the individual selection. By calculating the selection probability of each feature, then the selection probability can be obtained by the proportional fitness function to select the chromosomes left behind. The offspring left by the fitness function is then the first offspring. As we continue to obtain offspring, each offspring is a better solution than the previous generation. Then what will be obtained eventually is the optimal solution that cannot be obtained from the offspring. 


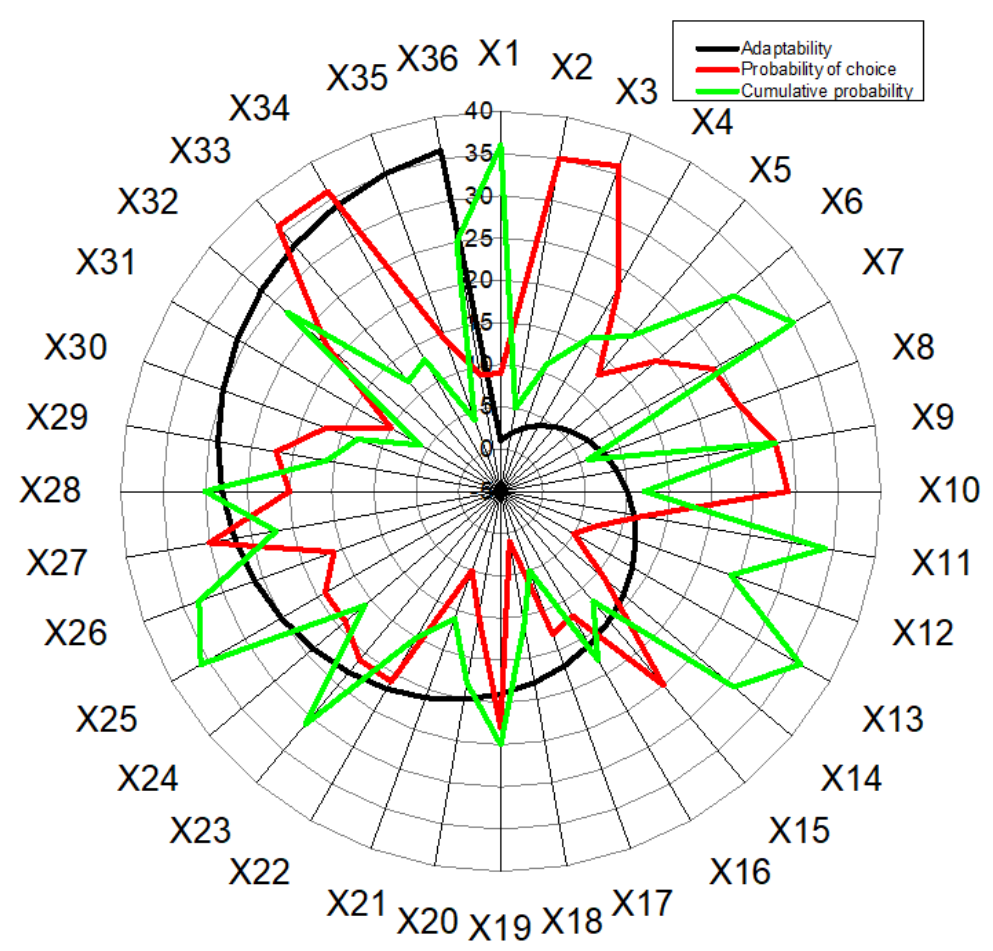

Figure 7 Selection probability

From the comparison of the whole convergence curves, the DE algorithm with better diversity has an advantage in text clustering in high dimensions and converges quickly to a good approximate optimal solution in the early stage of population update. Comparing the convergence curves of IDEPSO and DEPSO algorithms, the IDEPSO algorithm eventually converges to a stable F value better, and its convergence speed is faster than DEPSO, which means that it takes fewer iterations and running time to achieve a better optimal solution, which better reflects that the operation of adaptively adjusting the arrangement number of clustering centers can improve the efficiency of population update and running time. Comparing the convergence curves of each algorithm, it is easy to see that the IDEPSO algorithm makes full use of the features of both PSO and DE algorithms to obtain the optimal fitness value while maintaining a fast convergence rate, and has a strong optimization capability and efficient operation efficiency.

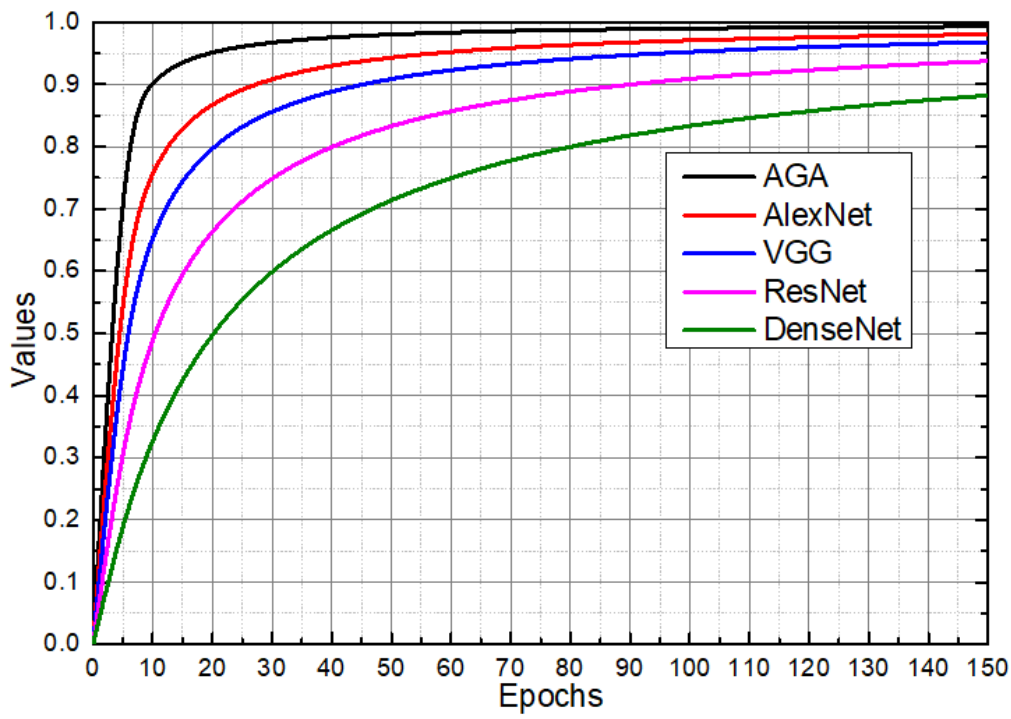

Figure 8 Average convergence curve

The average convergence curves of $\mathrm{F}$ for each algorithm on the four data sets are given in Figure 8 . From the first 30 iterations, the convergence curves of PSO and GQPSO algorithms on the four datasets are very steep, converge to a low stable value very early, converge the fastest, and obtain the adaptation value F. The GAI-PSO algorithm is relatively flat, and the F curve increases steadily, while the convergence curves of DE, 
DEPSO and the convergence curves of DE, DEPSO, and IDEPSO algorithms are close to each other and steeper but the converged $\mathrm{F}$ values are at a higher level, indicating that these algorithms can achieve better fitness values $\mathrm{F}$ in the early stage of population evolution. The IDEPSO algorithm converges almost completely to the highest fitness value F, except in the DS1 data set. After 60 iterations, GAI-PSO starts to converge and stabilize, while the other algorithms converge completely.

Then the parameter cases of the PSO algorithm and DE algorithm are tested on these datasets, and the parameter combinations that achieve the best text clustering results are obtained. Finally, the IDEPSO algorithm proposed in this paper is tested on the test dataset with PSO algorithm, DE algorithm, and other types of improved algorithms of PSO, and then the performance of these algorithms is compared in terms of internal evaluation metrics, convergence curves, external evaluation metrics, and stability to verify the effectiveness and feasibility of the proposed IDEPSO algorithm.

\section{Conclusion}

In the exploration of the crossover and variation problem, a new adaptive genetic operator is constructed based on the individual fitness between the average fitness and the maximum fitness, and the degree of concentration and dispersion of the fitness of the population in evolution, which non-linearly adaptively regulates the operational process of genetic evolution, and takes half of the maximum fitness value as the judgment criterion, avoiding the situation that the crossover probability and variation probability are zero values when the individual takes the maximum fitness value. It avoids premature convergence of the algorithm, ensures population diversity, and gives full play to the local search advantages of the crossover and variation operators. This paper introduces the background and significance of offline character recognition from the beginning. The current research status of handwritten character recognition is discussed, and then the basic principle and recognition process of offline Chinese character recognition are discussed. And an improved feature extraction algorithm is proposed for experiments. Finally, the improved character feature extraction algorithm is combined with a genetic algorithm to improve the recognition accuracy of feature extraction to a greater extent. The final experimental results are compared with the traditional feature extraction algorithm, and then the corresponding conclusions are drawn based on the results. The main content of the improved algorithm is to put the features obtained from the characters into the undirected graph, and then transform the character curve to the line, and compare each line of features obtained from the characters with the same set, and then use the least deviation method to complete the recognition. The whole process belongs to the category of structural features. The recognition speed is fast, the recognition accuracy is high, and it has high operability.

\section{Declarations}

Funding: This work is supported by the Project of Shandong Province Higher Educational Science and Technology Program: A Corpus-based Study on the Translation of Gaomi Dialect in English Versions of Mo Yan's Novels(No. J18RA238).

Conflicts of interests: None

Availability of data and material: Not applicable.

Code availability: Not applicable.

Authors' Contributions: Tang Xiaohui designed the model, collected dataset, performed the analysis, validated the results, written and reviewed the manuscript.

\section{References}

[1] Ahlawat S, Rishi R. A genetic algorithm based feature selection for handwritten digit recognition[J]. Recent Patents on Computer Science, 2019, 12(4): 304-316.

[2] Azmi S D, Kusumaningrum R. Relevance Feedback using Genetic Algorithm on Information Retrieval for Indonesian Language Documents[J]. Journal of Information Systems Engineering and Business Intelligence, 2019, 5(2): 171-182.

[3] Wang J, Qin J, Xiang X, et al. CAPTCHA recognition based on deep convolutional neural network[J]. Math. Biosci. Eng, 2019, 16(5): 5851-5861.

[4] Ratre A. Stochastic gradient descent-whale optimization algorithm-based deep convolutional neural network to crowd emotion understanding[J]. The Computer Journal, 2020, 63(2): 267-282.

[5] Xu J, Ding W, Zhao H. Based on Improved Edge Detection Algorithm for English Text Extraction and Restoration From Color Images[J]. IEEE Sensors Journal, 2020, 20(20): 11951-11958. 
[6] Kumar M, Jindal M K, Sharma R K, et al. Character and numeral recognition for non-Indic and Indic scripts: a survey[J]. Artificial Intelligence Review, 2019, 52(4): 2235-2261.

[7] Puri S, Singh S P. A fuzzy matching based image classification system for printed and handwritten text documents[J]. Journal of Information Technology Research (JITR), 2020, 13(2): 155-194.

[8] Elharrouss O, Almaadeed N, Al-Maadeed S, et al. Image inpainting: A review[J]. Neural Processing Letters, 2020, 51(2): 2007-2028.

[9] Virk I S, Maini R. Medical image segmentation based on fuzzy 2-partition Kapur entropy using fast recursive algorithm[J]. International Journal of Intelligent Engineering Informatics, 2020, 8(4): 346-373.

[10] Ziani A, Azizi N, Zenakhra D, et al. Combining RSS-SVM with genetic algorithm for Arabic opinions analysis[J]. International Journal of Intelligent Systems Technologies and Applications, 2019, 18(1-2): 152178.

[11] Pandey D, Pandey B K, Wairya S. Hybrid deep neural network with adaptive galactic swarm optimization for text extraction from scene images[J]. Soft Computing, 2021, 25(2): 1563-1580.

[12] Lizarraga A, Sprinkle J, Lysecky R. Automated Model-Based Optimization of Data-Adaptable Embedded Systems[J]. ACM Transactions on Embedded Computing Systems (TECS), 2020, 19(1): 1-22.

[13] Li X, Liu T, Li A, et al. Genetic polymorphisms and the independent evolution of major histocompatibility complex class II - DRB in sibling bat species Rhinolophus episcopus and Rhinolophus siamensis[J]. Journal of Zoological Systematics and Evolutionary Research, 2021, 59(4): 887-901.

[14] Feng Z, Sun P. Segmentacion de Imagenes Medicas Basada en la Red Neuronal Optimizada de GA[J]. Investigación Clínica, 2019, 60(1): 233-241.

[15] Kumar A, Jaiswal A. Swarm intelligence based optimal feature selection for enhanced predictive sentiment accuracy on twitter[J]. Multimedia Tools and Applications, 2019, 78(20): 29529-29553.

[16] Sen S, Mitra M, Bhattacharyya A, et al. Feature selection for recognition of online handwritten bangla characters[J]. Neural Processing Letters, 2019, 50(3): 2281-2304.

[17] Ma J, Xue B, Zhang M. A Hybrid Filter-Wrapper Feature Selection Approach for Authorship Attribution[J]. International Journal of Innovative Computing, Information and Control, 2019, 15(5): 1989-2006.

[18] Chouhan S S, Kaul A, Singh U P. Image segmentation using computational intelligence techniques[J]. Archives of Computational Methods in Engineering, 2019, 26(3): 533-596.

[19] Abasi A K, Khader A T, Al-Betar M A, et al. A novel ensemble statistical topic extraction method for scientific publications based on optimization clustering[J]. Multimedia Tools and Applications, 2021, 80(1): 37-82.

[20] Sanches S R R, Sementille A C, Aguilar I A, et al. Recommendations for evaluating the performance of background subtraction algorithms for surveillance systems[J]. Multimedia Tools and Applications, 2021, 80(3): 4421-4454.

[21] Biswas A, Islam M S. An Efficient CNN Model for Automated Digital Handwritten Digit Classification[J]. Journal of Information Systems Engineering and Business Intelligence, 2021, 7(1): 42-55.

[22] Bibi K, Naz S, Rehman A. Biometric signature authentication using machine learning techniques: Current trends, challenges and opportunities[J]. Multimedia Tools and Applications, 2020, 79(1): 289-340.

[23] Dutta S, Saha N, Das A K, et al. Clustering Model for Microblogging Sites using Dimension Reduction Techniques[J]. International Journal of Information System Modeling and Design (IJISMD), 2019, 10(2): $26-45$. 